\title{
Sensor Integration in Castings Made of Aluminum - New Approaches for Direct Sensor Integration in Aluminum High Pressure Die Casting
}

\author{
Rico Tiedemann ${ }^{1, a^{*}}$, Christoph Pille ${ }^{2, b}$, Gerrit Dumstorff ${ }^{1, c}$, Walter Lang ${ }^{1, d}$ \\ ${ }^{1}$ Institute for Microsensors, -Actuators and -Systems IMSAS, University Bremen, \\ Otto-Hahn-Allee, NW1, 28359 Bremen, Germany \\ ${ }^{2}$ Fraunhofer Institute for Manufacturing Technology and Advanced Materials IFAM, \\ Wiener Straße 12, 28359 Bremen, Germany \\ artiedemann@imsas.uni-bremen.de, ${ }^{\mathrm{b}}$ christoph.pille@ifam.fraunhofer.de, \\ cgdumstorff@imsas.uni-bremen.de, 'wlang@imsas.uni-bremen.de
}

Keywords: thick film sensors, structural health monitoring, high pressure die casting, material integrated sensing

\begin{abstract}
The use of sensors for detection, measurement and evaluation of mechanical and thermal loads is well known and essential for the implementation of »Structural Health Monitoring « (SHM). For this, sensors are mainly used for condition monitoring of mechanical loads and their impact to the castings state, which is a decisive advantage for safety-related components. The use of sensors on the surface of metallic components, in particular of cast metal components made of aluminum, is still limited to the use of strain gauges. They are usually applied on the surface of the cast metal components and get fixed by adhesives. The idea is, to integrate the sensors directly during the aluminum casting process. Since integrated sensors are naturally protected against chemical and mechanical influences, furthermore the load can be measured directly at the point of interest inside the component. Measurement data can be recorded and provide a good data basis for future calculations and dimensioning of components, which is known as »Data Mining " or »Industrial Data Space«. New technology and material combinations, which allow the fabrication of sensors capable of withstanding force and temperature during the integration process in aluminum casting, are investigated.

In this paper, the design and fabrication of a strain gauge printed on an aluminum sheet is shown. These sensor sheets get integrated in aluminum during high pressure die casting (HPDC) in a way that a specimen is build up. The specimen is characterized in a fatigue bending test and the sensor data was read permanently during this test. It is shown that the new approach with printed thick film sensors on aluminum substrate sheets works properly to withstand the heavy thermal conditions during high pressure die casting. The fabricated sensor is able to sense the mechanical tiredness and detects the fatigue of the metal matrix. This a first step to use such material integrated sensors in structural health monitoring applications.
\end{abstract}

\section{Introduction}

Future components in the automotive, railroad and aviation industry are being designed with regard to its fatigue strength, to achieve lower operating costs by weight optimization. To get a better basis of calculation, components are equipped with sensory functions to monitor the physical condition of critical parts [1]. The new approach is to equip load-bearing components especially for automotive and mobile applications with integrated sensors and simple evaluation electronics for structural health monitoring (SHM). With integrated sensors and continously recording of the mechanical load, »Data Mining《 is one method to characterize the current damage state and provides a good wear indicator for structural components. This wear indicator is often more expressive for dynamical and unpredictably stressed components than the implied wear by the overall mileage in cars. It will be possible to classify smaller accidents as well as peak-loads in structural components and chassis. With advanced data analytic technics significant improvement in the prediction of fatigue strength accuracy over previous efforts could be achieved $[2,3]$. Through integrated sensors 
haptic feedback of the steering in cars with "steer by wire" systems can be improved. Another possible application is an exoskeleton or an industrial robot with integrated sensory function to make a human-machine interaction as safe as possible. External sensors are applied to the surface of components, whereas internal sensors are enclosed by the component. Therefore, external sensors like strain gauges or temperature sensors, are exposed to harsh environments and have to be protected against corrosive conditions, contamination or damage. Furthermore, with surface mounted sensors a model is required to get physical quantities from the inner of the component, because the data is measured at the surface. External sensors, like bonded strain gauges show creepage trough two mechanisms. On one hand the spring effect between the measuring grid and the carrier film and on the other hand the holding force between the carrier film and the adhesive [4]. Both effects can be reduced through direct integration of the sensor element into the cast matrix without the use of adhesives. Integration of sensors in cast aluminum is a challenging task, caused by the high temperature of $\sim 700{ }^{\circ} \mathrm{C}$ of molten aluminum. Additionally heavy mechanical loads in high pressure die casting caused by high filling velocity of $\sim 20-40 \mathrm{~m} / \mathrm{s}$ and afterwards redensification phase are critical impacts to the sensor.

Strain gauges are fabricated in thin and thick film technology. Common thin film sensors are silicon-based and critical in use for embedding during casting with high failure rates, due to the different elongation coefficients of silicon $\left(\alpha_{\mathrm{Si}}=2.6 \mathrm{E}-61 / \mathrm{K}\right)$ and aluminum $\left(\alpha_{\mathrm{Al}}=21 \mathrm{E}-61 / \mathrm{K}\right)$, hence high compressive stress is induced in the silicon while cooling phase. This stress causes cracks in the silicon substrate, or leads to delamination of the functional layers, both leads to failure [5]. On the other hand, compressive stress can be an advantage to realize a shape connection between the sensor and the metal matrix. Even better would be a completely positive material joint. Aluminum as substrate for thick film sensors, fabricated by screen printing can be used. The natural aluminumoxide layer prevents a positive material joint. In [6] A199.5 sheets are integrated via high pressure die casting (HPDC), which are pre-treated and dipped into zincate solution, which makes a positive material joint possible.

Any integrated sensor has effects on the properties of the component in which it is integrated in. The mechanical strength and thermal conductivity changes with the properties of the integrated sensor $[7,8,9]$. Widely varying E-Modules can cause stress in the aluminum matrix under load, which weakens the component. This foreign body effect through thermal and mechanical influences has to be minimized, therefore in a new approach aluminum is used as substrate instead of silicone. The induced thermal stress through different elongation coefficients is reduced to a minimum, same applies to the thermal conductivity.

\section{Thick film sensor fabrication on aluminum substrate}

The screen printing process is a proven method for the fabrication of sensors and electronic components like SMD resistors. A common substrate is aluminum oxide. For the presented strain gauge sensors conductor, resistor and dielectric thick film pastes are used, which are designed to build electric circuits directly onto aluminum substrate [10]. Each layer is processed by screen printing and gets individually fired. The general structure of the sensors can be seen in fig. 1 . The layers, which are applied on aluminum sheets as substrate, are in total thinner than $200 \mu \mathrm{m}$. 


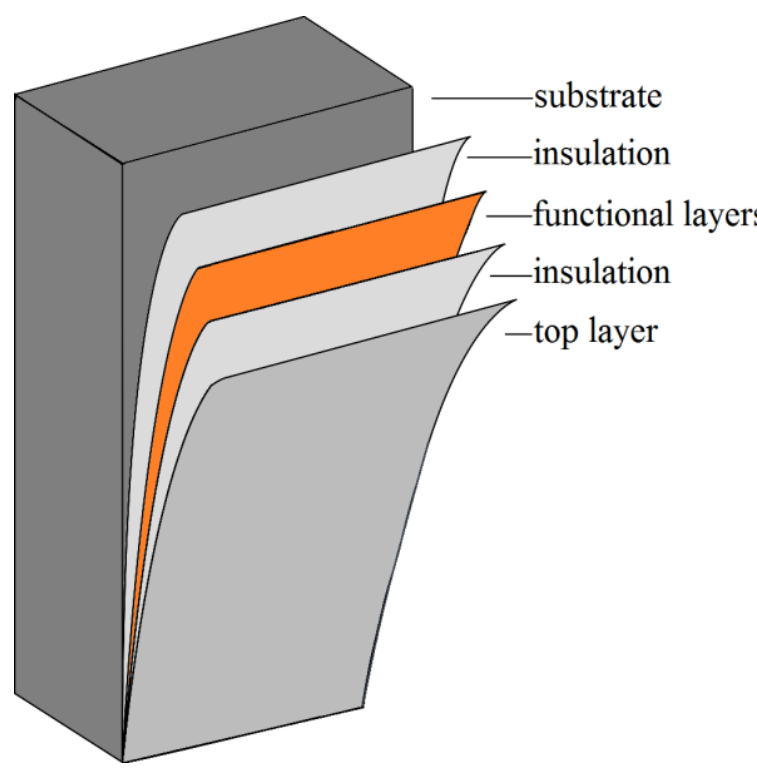

Fig. 1. General structure of the sensors

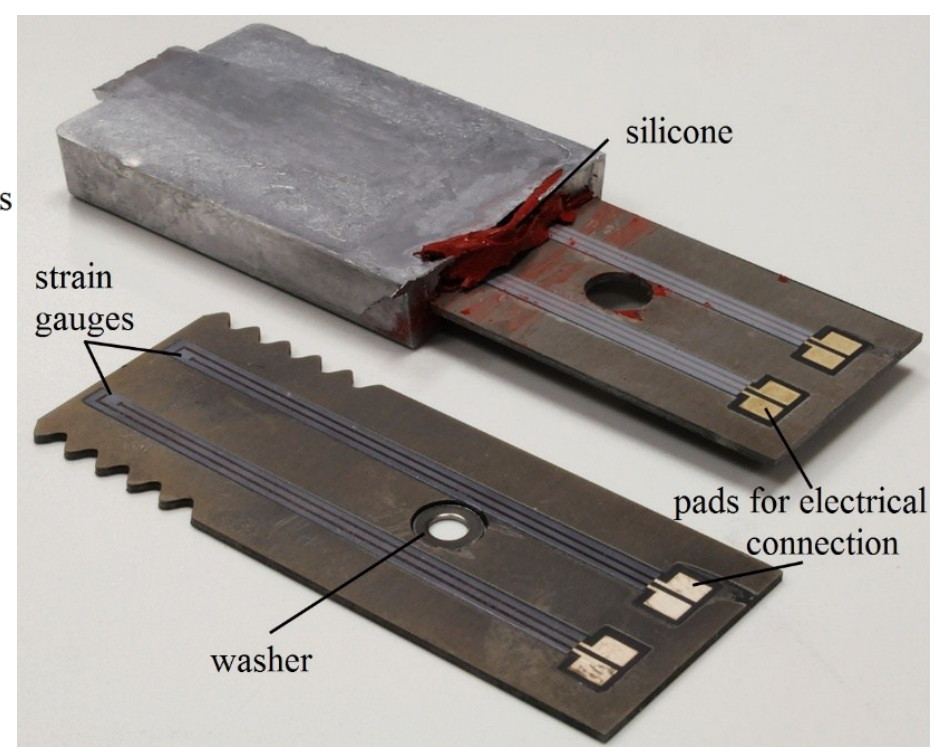

Fig. 2. Processed sensor before and after integration

With this design, the general feasibility of direct integration in aluminum casting will be proved. Different top layers were tested to minimize the impact of the molten aluminum to the functional layers. Sensors with printed and fired insulation top layer were tested as well as sensors with an additional printed but unfired insulation top layer. Furthermore, sensors with an additional top layer of high temperature stable silicone were tested. In fig. 2, a processed sensor on an AlMg3 substrate before and after integration can be seen. To protect the sensor from the locking force of the HPDC machine, every sensor got a small protection layer of high temperature stable silicone in the area of clamping. The washer in the substrate is used to fix the sensor sheet in the mold of the HPDC machine, which provides a magnet in the cavity of the die to mount different ferromagnetic materials.

\section{Sensor integration during high pressure die casting}

The presented thick film sensors on aluminum substrates are successfully integrated during high pressure die casting, illustrated in fig. 3. Short casting cycles in HPDC brings cost efficiency for large quantities, yet also harsh conditions with up to 800 bar redensification pressure and a high inrushing velocity of the melt. The key benefit of HPDC for the sensor is the short exposure time to high temperatures. In HPDC the mold is actively tempered, hence the molten aluminum is cooled down fast, reaching a uncritical temperature below $\sim 300{ }^{\circ} \mathrm{C}$ within a few seconds. As alloy the common standard AlSi9MgMn (Silafont 36) is used. 


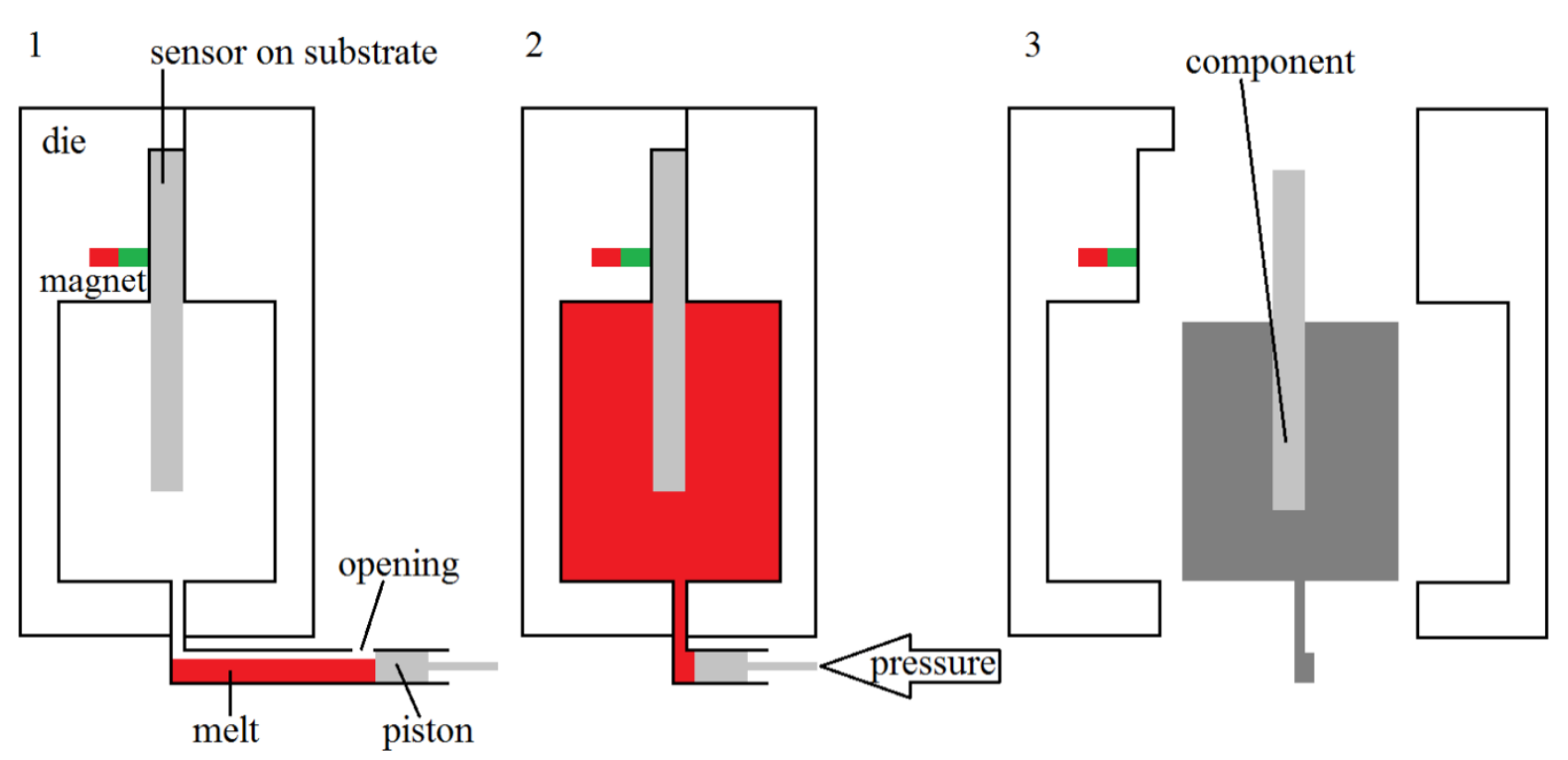

Fig. 3. High pressure die casting cycle with integrated strain gauge sensor; 1 : inserting the sensor in the mold and closing the mold with a locking force of $\sim 6,000 \mathrm{kN}$, additional the cavity is evacuated to partial vacuum before casting; 2: filling of the cavity with redensification pressures of up to 800 bar, afterwards 10 to 20 s cooling phase through active oil tempering; 3: ejecting of the component and coating of the mold for the next cycle

From the tested top layers, a dried but unfired additional insulation layer as top layer increased the yield to $75 \%$ in total and nine out of twelve sensors survived the integration process.

\section{Fatigue bending test setup}

Fatigue bending tests with the above described, fabricated and integrated sensor were made to gain information about the properties of the strain gauge, such as hysteresis, sensordrift and durability. To read out the sensor, it is arranged in a quarter bridge with an instrumentational amplifier as shown in fig. 4. In fig. 5 the component can be seen in the 3-point bending device. The pressure swell test was made, using the universal testing machine (DYNA-MESS TP $10 \mathrm{HF}$ ) with an alterning load amplitude of $3,000 \mathrm{~N}$ at a mean load value of $5,000 \mathrm{~N}$, which leads to a maximum stress at the sample of $164.5 \mathrm{~N} / \mathrm{mm}^{2}$ and a minimum stress of $41.1 \mathrm{~N} / \mathrm{mm}^{2}$. The stress applied on the sensor depends on its position to the neutral fiber. Within this specimen the sensor is $1.75 \mathrm{~mm}$ out of the neutral fiber, hence the sensor is set to stress from $60 \mathrm{~N} / \mathrm{mm}^{2}$ to $15 \mathrm{~N} / \mathrm{mm}^{2}$. The test frequency is set to $2 \mathrm{~Hz}$.

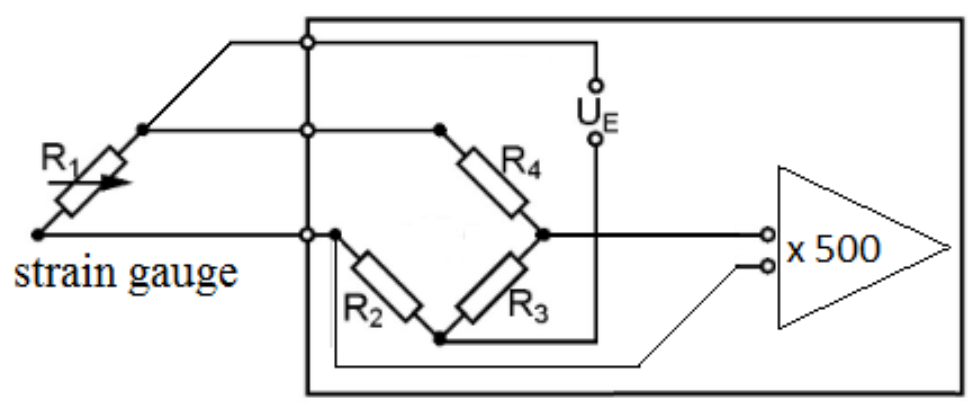

Fig. 4. Quarter bridge with an instrumentational amplifier 


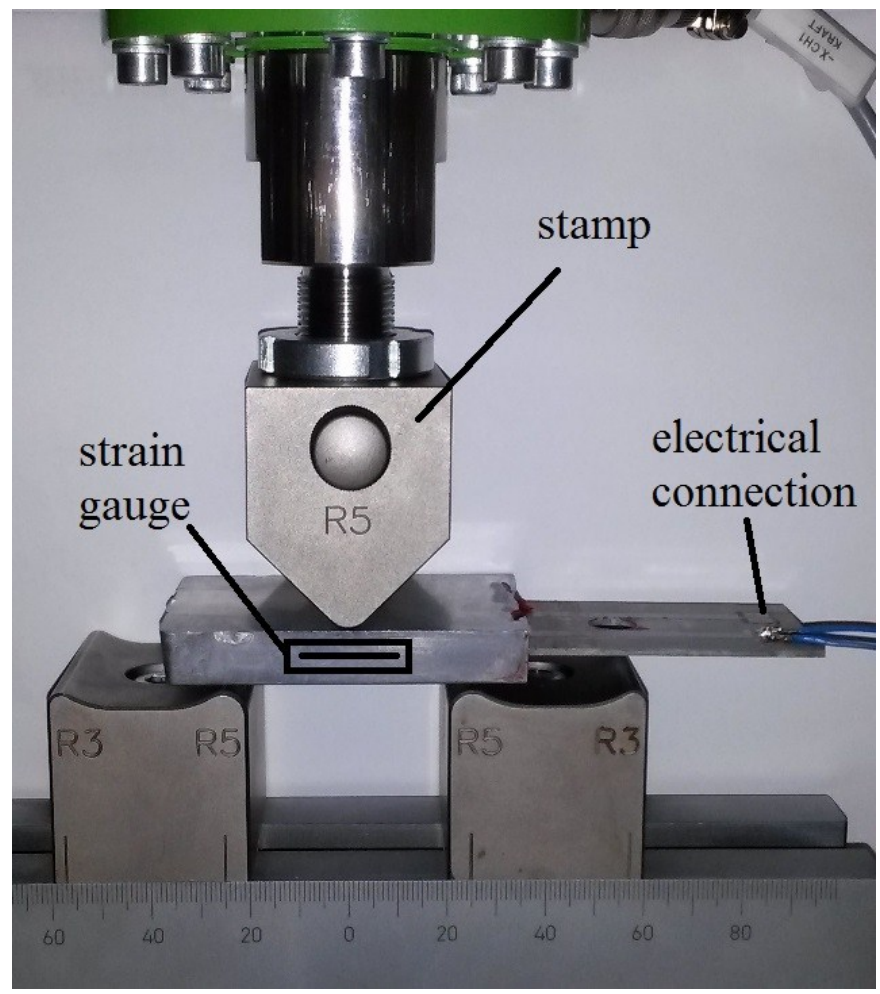

Fig. 5. 3-point-bending-device with inserted specimen

\section{Results and discussion}

The durability of the sensor exceeds the durability of the component, therefore the fatigue can be recorded. Fig. 6 shows the destroyed component after the fatigue test in the 3-point bending device.

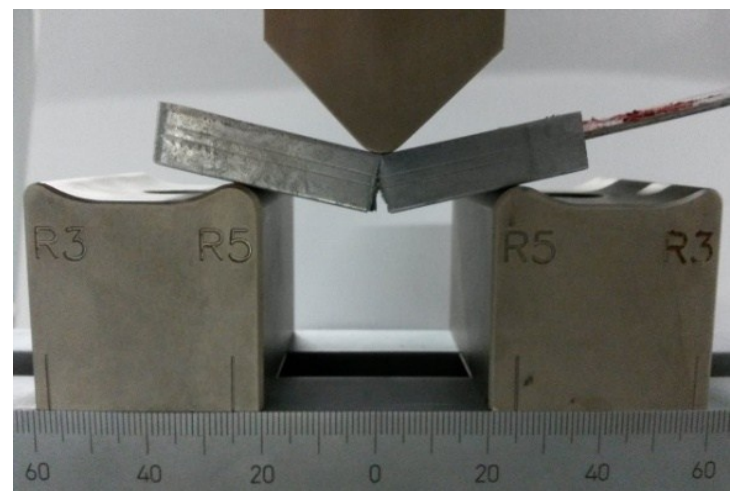

Fig. 6. Destroyed component after fatigue test

The trend of the fatigue test can be seen in fig. 7. The bottom curve shows a typical characteristic for an aluminum fatigue test. The sample including the sensor shows innitial changes of the structure along the first 60,000 cycles, due to the motion of dislocations at the beginning. Along the next 550,000 cycles dislocation accumulations leads to internal stress. If the internal stress exceeds the cohesion strength microcracks occure and grow. Growing microcracks lead to a decreasing cross-section and thus an increas in stress, resulting in a failure of the sample. The sensor is set to tensile stress from $60 \mathrm{~N} / \mathrm{mm}^{2}$ to $15 \mathrm{~N} / \mathrm{mm}^{2}$ over 710,000 times and can be used as fatigue indicator. 


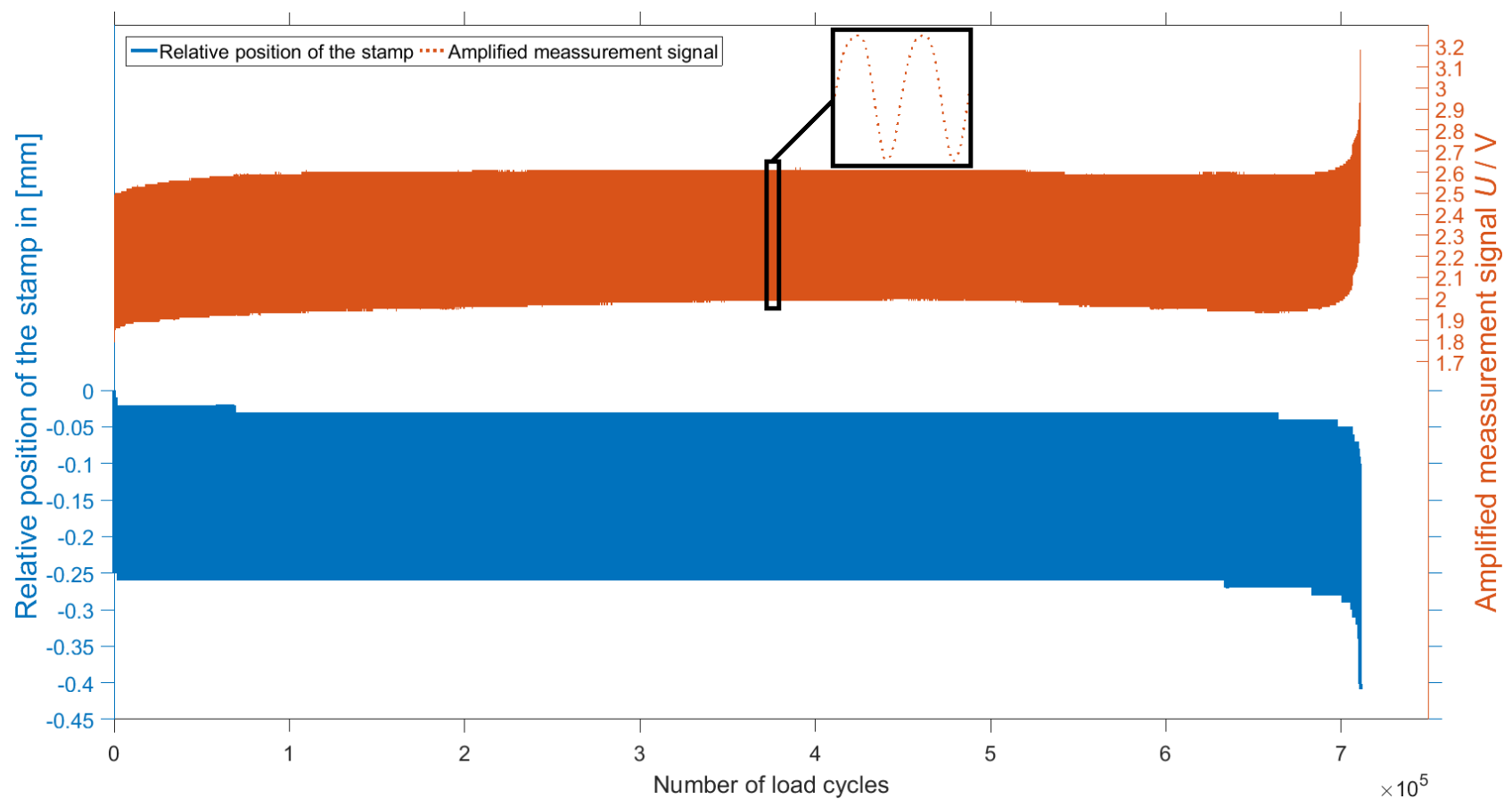

Fig. 7. Result of the fatigue test

Fig. 8 shows the last 12,000 bending cycles, where microcracks and therefore the mechanical load increases due to decreasing cross-section. The resistance of the strain gauge is changing with increasing load per remaining cross-section, which can be seen in the upper curve of fig. 8. At 706,000 load cycles, the stress of a large accumulation of dislocations exceeds the cohesion strength, and a macroscopic cracks occures. The load must be supported by the remaining crosssection, wich can be seen in the fast rise of the meassurement signal. The sensor is able to detect the fatigue of the component and can be used as an early-warning predictive system. Further investigations will show, if the fatigue can be seen in the sensor signal before microscopic craks occure on the surface of the component.

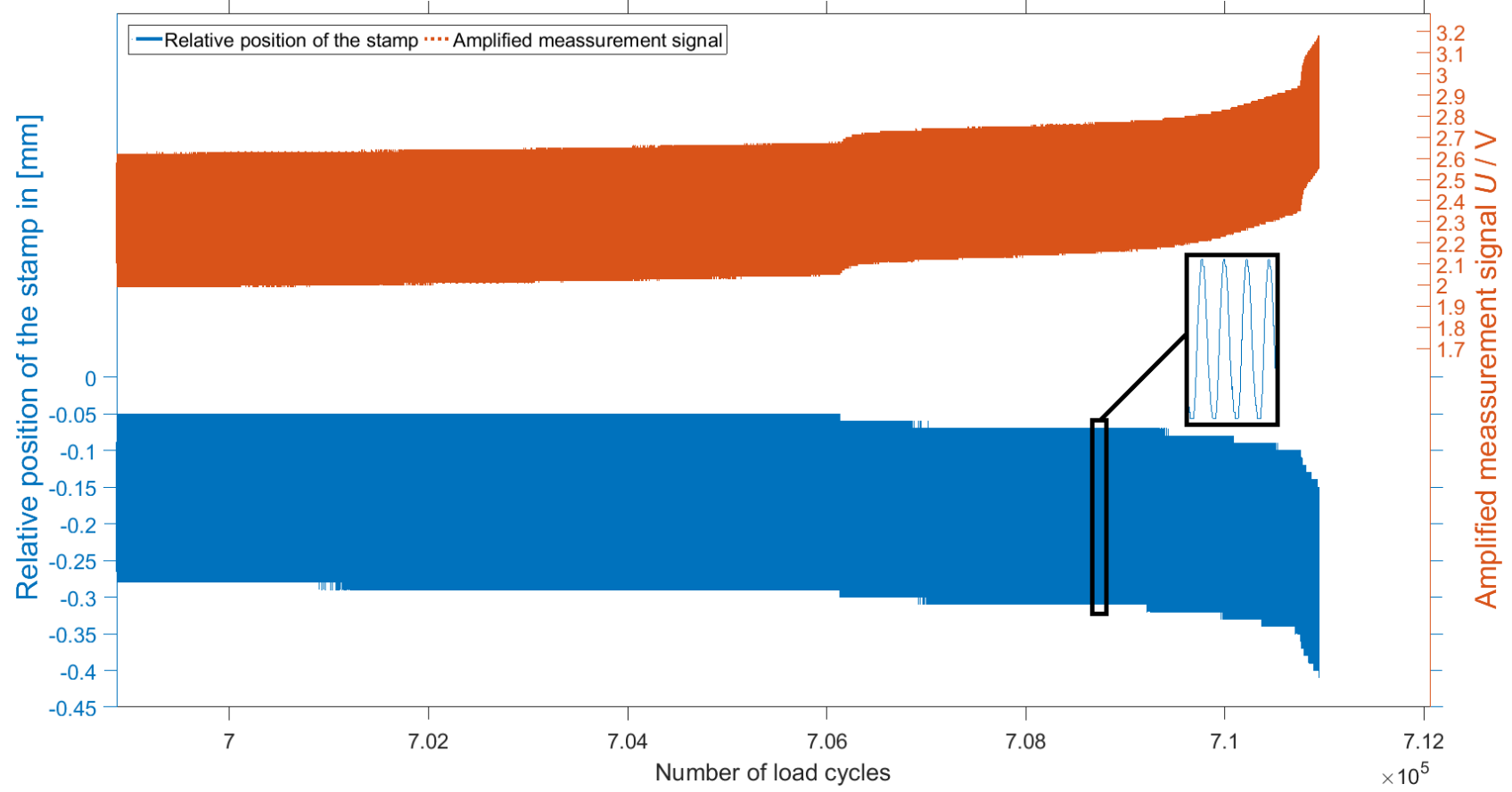

Fig. 8. Enlarged section of the fatigue test with failure 


\section{Conclusion and Outlook}

A strain gauge is presented that was directly printed on aluminum substrate for the integration in an aluminum casting matrix during HPDC. A significant number of strain gauges survived the HPDC process. We have proved that measuring strain with integrated sensors in aluminum cast is possible. An initial result of long-term behaviour has been presented. The durability of the integrated sensor exceeds the durability of the component and the fatigue of the component can be measured. With »Data Mining « it might be able to estimate the fatigue of the component by damage accumulation over time as a good wear indicator for structural components. This is a first step to use these material integrated sensors in structural health monitoring applications.

In further investigations, the influence of the foreign body effect through the integrated sensors in cast aluminum components has to be analysed. Specimen with integrated sensors have to be compared with specimen without integrated sensors. To find out about the mechanical properties Wöhler fatigue tests have to be made. In addition different casting parameters and their influences respectively their impact on the sensor have to be investigated, such as various mold filling velocities, various solidification pressures or a subsequent heat treatment of the castings.

\section{Acknowledgement}

We would like to thank the Deutsche Forschungsgemeinschaft / German Research Foundation (DFG) for funding the project »SINA - Sensorintegration in Aluminiumguss « (Förderkennzeichen BU 1796/12-1, LA 1471/18-1) and the University of Bremen for funding the postdoctoral research project $»$ AddSense - Additive manufacturing for local fabrication of sensor structures for material integrated sensing $\ll$.

\section{References}

[1] J. Gobrecht, Werkstofftechnik - Metalle, Oldenbourg Verlag, 2006

[2] A. Agrawal, P. D. Deshpande, A. Cecen, G. P. Basavarsu, A. N. Choudhary and S. R. Kalidindi, Exploration of data science techniques to predict fatigue strength of steel from composition and processing parameters, Integrating Materials and Manufacturing Innovation, vol. 3:8, (2014) 1-19

[3] J.M. Schoolin, M. Brown and P.A.S. Reed, An example of the use of neural computing techniques in materials science-the modelling of fatigue thresholds in Ni-base superalloys, Material Science and Engineering, vol. A 260, (1999) 222-39

[4] H.-R. Tränkler and E. Obermeier, Sensortechnik, Handbuch für Praxis und Wissenschaft, Springer Verlag, 1998

[5] G. Dumstorff and W. Lang, Resistive silicon microstructure for embedding in aluminium during casting, IEEE/ASME International Conference on Advanced Intelligent Mechatronics, (2014) $582-586$

[6] M. Schwankl, M. Rübner, R.F. Singer and C. Körner, Integration of PZT-ceramic modules using hybrid structures in high pressure die casting, Procedia Materials Science, vol. 2, (2013) 166-172

[7] G. Dumstorff and W. Lang, Investigations on the impact of material integrated sensors with the help of fem-based modelling, Sensors, vol. 15, no. 2, (2015) 2336-2353

[8] G. Dumstorff and W. Lang, Failure of silicon substrates embedded in epoxy resin, Procedia Technology, vol. 15, (2014) 216-220

[9] M. K. Moghaddam, D. Boll and W. Lang, Embedding rigid and flexible inlays in carbon fiber reinforced plastics, IEEE/ASME International Conference, 2014

[10] Fascination Light - Smart Materials for LED Lighting, (Online 23.01.2017), Information on http://www.heraeus.com/media/media/het/doc_het/products_and_solutions_het_documents/ thick_film/LED_Brochure.pdf 